\title{
Lieux et non-lieux de Troie
}

\section{David Bouvier}

\section{(2) OpenEdition}

Journals

Édition électronique

URL : http://journals.openedition.org/edl/275

DOI : $10.4000 /$ edl. 275

ISSN : 2296-5084

\section{Éditeur}

Université de Lausanne

\section{Édition imprimée}

Date de publication : 15 mai 2010

Pagination : 9-38

ISBN : 978-2-940331-22-2

ISSN : 0014-2026

\section{Référence électronique}

David Bouvier, «Lieux et non-lieux de Troie », Études de lettres [En ligne], 1-2 | 2010, mis en ligne le 15 mai 2013, consulté le 21 décembre 2020. URL : http://journals.openedition.org/edl/275 ; DOI : https:// doi.org/10.4000/edl.275 


\section{LIEUX ET NON-LIEUX DE TROIE}

Il y a des débats sans fin. Les récentes fouilles menées sur le site de Troie ont relancé une fois encore la fameuse question homérique, tandis que l'Iliade a reçu, en 2004, une nouvelle actualité par le biais du cinéma hollywoodien. La guerre de Troie et ses héros ont-ils existé? Homère est-il un témoin fiable? La question remonte à l'Antiquité. Mais les formulations et les réponses n'ont cessé de varier au cours des siècles. A mieux y regarder - et si l'on en retrace l'histoire - la question de la guerre de Troie n'existe qu'en fonction de thèses et d'enjeux qui dépassent largement la seule compréhension et interprétation de l'Iliade. L'historien de la tradition classique peut se promener dans le temps pour vérifier comment chacun réinvente la coupure entre le mythe et la réalité historique. Entre l'Occident et l'Orient, Troie est un miroir d'intérêts divers, d'identités croisées, ce lieu où l'histoire peut enrichir le mythe pour mieux le récupérer ensuite. L'opposition du mythe et de la réalité n'est pas toujours la même.

\section{Quand le cinéma s'intéresse à l'archéologie}

Les coïncidences sont rares. En 2004, alors qu'éclate le scandale des prisons d'Abou Ghraib et que la guerre de l'Irak paraît toujours plus absurde $^{1}$, le festival de Cannes se réjouit de présenter, hors compétition, le film Troy de Wolfgang Petersen, distribué par la Warner Bros, avec dans le rôle d'Achille l'acteur Brad Pitt ${ }^{2}$. Dira-t-on que le cinéma se réapproprie l'interprétation des grandes œuvres classiques sur la guerre

I. Je renvoie ici à ma note «Des Fontaines de Troie à la prison d'Abou Ghraib». Mes vifs remerciements à Boghos Levon Zekiyan qui a discuté avec moi plusieurs points de ce travail, à Hans-Lukas Kieser qui m’a aidé pour traduire les articles parus en turc dans le journal Radikal et à Aurélie Matthey qui a relu ces pages.

2. Sur ce film, cf. par exemple J. Latacz, "From Homer's Troy to Petersen's Troy», et plus généralement M. M. Winkler (ed.), Troy: from Homer's Iliad to Hollywood epic. 
alors même que l'Amérique de G. W. Bush doute toujours plus de l'utilité et du bon sens de ses propres expéditions militaires? Que l'Iliade, ce "poème de la force " ${ }^{3}$, réinterprétée et mise en scène, peut servir à expliquer ou à justifier une guerre contemporaine? Mais plus qu'à cette question, que je pose en passant, je voudrais m'intéresser ici aux raisons du retentissant écho obtenu dans la presse et les médias par un film sur la guerre de Troie alors que l'Iliade n'est plus guère lue que par une minorité; aux raisons aussi qui ont pu conduire des hommes d'affaires à investir des capitaux conséquents dans l'énorme campagne publicitaire d'un film sur un tel sujet. Dans les années cinquante et soixante, les films qui avaient évoqué la guerre de Troie n'avaient rencontré qu'un succès très discret: l'Hélène de Troie de Robert Wise ${ }^{4}$, boudé par le public, est pourtant un beau film apprécié des connaisseurs. Et qui se souvient aujourd'hui de La Guerre de Troie de Giorgio Ferroni, sur les écrans en 1961, ou de La Colère d'Achille de Mario Girolami en 1962 ? 5

Les producteurs hollywoodiens ne sont pas des investisseurs naïfs et des études de marché ont bien sûr précédé la réalisation du film. Lorsqu'elle décide de promouvoir un film sur la guerre de Troie, la Warner Bros n'ignore pas que le débat scientifique sur l'authenticité de cette guerre a été relancé par les résultats d'une récente campagne de fouilles archéologiques sur le site de Troie (Hisarlik). Mieux encore: les médias se sont passionnés pour la question. Au début des années 2000, journaux, radios et télévisions donnent un très grand écho aux récentes découvertes des fouilles conduites, depuis 1988, par Manfred Korfmann - découvertes que le film ne manquera pas d'exploiter ${ }^{6}$. Avant donc que les rubriques de cinéma ne s'intéressent au personnage d'Achille incarné par Brad Pitt, Troie avait déjà fait la «une» des plus grands quotidiens:

3. Pour reprendre une expression de S. Weil, «L'Iliade ou le poème de la force (19401941)».

4. En 1956, film également distribué par la Warner Bros.

5. Sur ces films, cf. J. Solomon, "Viewing Troy», p. 96-97 et M. M. Winkler, "The Trojan War on the Screen", p. 207 (avec une erreur sur la date de sortie du film de Ferroni); cf. aussi sur l'Iliade et le cinéma, M. M. Winkler, "The Iliad and the Cinema».

6. En 1997 et 1998, les fouilles mettent au jour, dans la ville basse, un système complexe de souterrains pour l'approvisionnement en eau, souterrains que l'on retrouve dans le film de Petersen, alors qu'ils sont ignorés de la tradition homérique; je ne crois pas que l'on puisse tirer parti dans ce sens de la description des fontaines de Troie en Iliade, XXII, 153-157. Cf. M. Korfmann, N. Frank et A. Mangini, «Eingang in die Unterwelt. Die Höhle von Troia une ihre Datierung». 
couverture du Spiegel en 1998, trente pages dans l'édition américaine de National Geographic en 1999, dossier entier dans Das Bild le 17 février 2000, articles à répétition en 1993, 1996 et 1997 dans le New York Times, pour ne citer que ceux-là. Tout cela avant même la grande exposition sur Troie, inaugurée en mars 2001 et qui attirera en Allemagne et ailleurs près d'un million de visiteurs ${ }^{7}$. Mais que s'est-il donc passé pour que la presse et le public s'intéressent tant à l'Iliade et aux fouilles de Troie? Quelle pierre ou quel texte a-t-on trouvé qui a relancé le débat?

\section{L'énigme de Troie: nouvelle formulation}

Impossible ici de reprendre l'histoire des fouilles de Troie ${ }^{8}$; histoire qu'il faudrait compléter par celle des représentations imaginaires de cette cité 9 . Rappelons pourtant la part de l'enthousiasme et du scepticisme dans cette histoire. Enthousiasme d'Heinrich Schliemann qui commence ses fouilles à Troie en 1870 et découvre des objets précieux, en or et en argent (bijoux, vases), convaincu qu'il s'agit du trésor de Priam, le vieux roi de Troie chanté par l'Iliade. Mais le trésor provient du niveau II et doit être daté du Bronze ancien, c'est-à-dire vers 2500 av. J.-C. : plus de 1'000 ans d'écart avec la datation présumée de la guerre de Troie. Peu avant sa mort, Schliemann perçoit son erreur. Les archéologues, qui se succèdent ensuite sur le site, s'intéressent davantage aux couches supérieures, susceptibles de correspondre à l'époque des héros homériques. A la veille de la Seconde Guerre mondiale, Carl Blegen identifiait la Troie

7. Voir le catalogue: Troia: Traum und Wirklichkeit.

8. Le résultat des fouilles a été régulièrement publié dans Studia Troica dès le numéro 1, paru en 1991. Pour une synthèse des résultats récents et leur interprétation, cf. M. Korfmann, "Ilios, ca 1200 BC - Ilion, ca 700 BC». Voir aussi le catalogue de l'exposition Troia: Traum und Wirklichkeit et M. Korfmann (hrsg.), Troia: Archäologie eines Siedlungshügels und seiner Landschaft. L'interprétation de Korfmann a trouvé une caution philologique dans la présentation générale de J. Latacz, Troia und Homer: der Weg zur Lösung eines Rätsels. Pour d'autres points de vue, cf. J. P. Crielaard, «Homer, History and Archaeology: Some Remarks on Date of the Homeric World»; D. Hertel, Die Mauern von Troia: Mythos und Geschichte im antiken Ilion et id., Troia: Archäologie, Geschichte, Mythos. Le problème est bien formulé par F. Montanari, «Troia omerica e Troia anatolica. L'eterno dubbio tra realta e fantasia» et plus récemment par M. Benzi, «La guerre fantôme. La guerre de Troie dans une perspective archéologique».

9. Voir ici G. Danek, "The Story of Troy through the Centuries». 
d'Homère aux ruines découvertes sur le niveau de Troie VIIa détruite par un incendie vers 1180 av. J.-C.; mais ces ruines révélaient une petite cité et le scepticisme s'est installé: le petit bourg ne pouvait être la Troie d'Homère ${ }^{10}$. En réaction à l'enthousiasme de Schliemann, beaucoup ont postulé alors que Troie n'était qu'une invention poétique et la guerre des héros une légende.

En 1988, les fouilles sont cependant reprises avec des moyens nouveaux par Manfred Korfmann, professeur d'archéologie classique à l'Université de Tübingen et décédé en 2005, alors qu'il dirigeait encore les fouilles. A son arrivée à Troie en 1988, Korfmann dit vouloir rompre avec une tradition qui ne s'était intéressée au site que pour y trouver les preuves de l'authenticité de l'Iliade et qui voulait - c'était le rêve tout romantique de Schliemann - rendre vraie la légende: «Nous ne nous proposons pas, comme Schliemann le fit, de considérer l'Iliade comme la "vérité de l'évangile" ("gospel truth”)»" ${ }^{11}$. Intention louable, mais Korfmann ne va pas véritablement rompre avec la tradition inaugurée par Schliemann: celle d'une archéologie qui invente l'Homère dont elle a besoin.

Dès les premières semaines de fouilles, des mesures géomagnétiques permettent à l'archéologue de Tübingen de découvrir au pied de la citadelle de Troie une ville basse importante, de 270’000 mètres carrés, qui a pu abriter aux XIII'-XII e siècles une population de 7'000 à 10'000 personnes. Une telle population est considérable pour l'époque ${ }^{12}$. En 1995 , l'archéologue Donald Easton fait à son tour une découverte qui va se révéler fondamentale. Dans la ville basse, il découvre un sceau ${ }^{13}$ qui porte, sur ses deux faces, une inscription de langue louvite en hiéroglyphes hittites; c'est alors la plus ancienne inscription découverte sur

Io. Voir sur cet historique des fouilles, K. Reber, «Troie. Découverte et état actuel des connaissances", p. 15-17.

II. M. Korfmann, «Ilios, ca 1200 BC - Ilion, ca 700 BC», p. 211 (je traduis).

I2. Ces chiffres et leur interprétation ont très vite été critiqués avec virulence par Frank Kolb, professeur d'histoire ancienne et collègue de Manfred Korfmann à l'Université de Tübingen; cf. p. ex. Frank Kolb, "Troy VI : a trading center and commercial city». Mais, si l'on peut discuter l'importance et l'étendue de la ville basse, on ne peut remettre en question l'importance de la découverte de Korfmann dont l'interprétation s'est imposée. Relevons cependant que l'Iliade ignore l'idée d'une ville basse (cf. M. Benzi, «La guerre fantôme», p. 457).

13. Sur ce sceau qui va devenir le symbole des fouilles, cf. J. D. Hawkins et D. F. Easton, "A Hieroglyphic Seaal from Troia». 
le site. Peut-être utilisé dans un rituel funéraire, ce sceau pourrait révéler que Troie abritait, à cette époque, une population de culture anatolienne, comme l'indique par ailleurs les systèmes de fortification et d'autres données ${ }^{14}$. Si le site de Troie correspond à la cité décrite par l'Iliade, cela signifierait donc que la guerre des Achéens et des Troyens opposait deux cultures distinctes. D'aucuns s'attendaient à découvrir que les habitants de Troie étaient une colonie grecque, comme il en existait sur la côte anatolienne.

L'un des grands mérites de Korfmann fut alors de s'intéresser de plus près aux archives hittites retrouvées à Bogazköy/Hattusa, l'ancienne capitale des Hittites. Ces archives, qui contiennent de nombreux traités avec les puissances voisines, sont essentielles et permettent de reconstituer l'histoire du II ${ }^{\mathrm{e}}$ millénaire av. J.-C. sur le territoire de la Turquie. E. Forrer, en 1924 déjà, avait proposé d'identifier les Ahhiyawa mentionnées dans les textes hittites aux Achéens/Mycéniens. De nouveaux textes et une meilleure lecture permet aujourd'hui de confirmer, avec une grande probabilité la thèse de Forrer. Si Homère ne mentionne pas les Hittites, les textes hittites connaissaient le monde achéen et plusieurs noms sont cités qui pourraient être rapprochés - et le débat se poursuit - de noms de dieux ou de héros grecs ou troyens mentionnés dans l'Iliade: Alaksandu/Alexandre, Appaliunaas/Apollon, Attarissyia/Atrée, Pijamaradu/Priam, Tawaglawa/Etéocle, etc.

Mais surtout, en août 2003, Frank Starke a offert une interprétation plus précise d'un texte hittite connu depuis longtemps (KUB XXVI.91). Il l'interprète comme une lettre envoyée par un roi des Ahhiyawa au roi hittite Hattusili III (1267-1237 av. J.-C.). Cette lettre évoque une île en face de Wilusa, que les Ahhiyawa réclament. D’autres documents permettent de situer cette lettre dans un contexte historique plus précis. Ils laissent comprendre que Wilusa est située dans le nord-ouest de l'Anatolie; elle était une petite seigneurie dépendant de l'empire hittite qui tenait à ses alliés ou vassaux de l'Anatolie occidentale. Un texte garde notamment la trace d'un traité entre le roi hittite Muwattali (1295-1272 av. J.-C.) et le roi de Wilusa, Alaksandu (équivalent hittite

I4. Sur la moindre importance de ce sceau, cf. T. Bryce, «An historian's observation on Troy and homeric tradition", p. 34. Même s'il est enclin à penser que la population de Troie est louvite, Bryce ne donne au sceau, qui reste une pièce unique, qu'une valeur d'« interesting curiosity». 
du nom d'Alexandre) ${ }^{15}$. Ces données s'accordent parfaitement avec la possibilité d'une expédition au XIII ${ }^{\mathrm{e}}$ siècle des Ahhiyawa contre le petit royaume de Wilusa, possibilité qui relance complètement le débat sur un arrière-fond historique de l'Iliade.

Car toutes les données convergent pour confirmer désormais la vieille intuition de P. Kretschmer que Wilusa est bien la Troie de l'Iliade. Pour la première fois, on a des arguments solides, définitifs diront certains, pour penser que la ville de Troie évoquée par Homère est une réalité et non une invention poétique. Et si le nom d'Alexandre était un souvenir homérique du roi Alaksandu? On aurait un argument de plus pour supposer, cette fois, l'historicité de la guerre de Troie. Pour Korfmann suivi par J. Latacz, le contexte politique est clair et cohérent. A la fin du XIII ${ }^{\mathrm{e}}$ siècle, la tension entre les Ahhiyawa et Wilusa semble avoir été à son comble et l'on peut noter que les murailles de Troie ont été à cette époque-là renforcées. Si l'on ajoute à ce tableau les données qui évoquent, au XIII ${ }^{\mathrm{e}}$ siècle toujours, la prise de la cité de Milet, jusque-là achéenne, par la puissance hittite, on comprend que les Achéens/Ahhiyawa aient pu être tentés de compenser cette perte par l'occupation d'un autre site qui pouvait leur assurer une voie vers l'Est. Une guerre de Troie à la fin du XIII ou au début du XII e siècle est une hypothèse plausible. Du moins, des savants comme Korfmann et Latacz veulent-ils y croire!

L'incontestable progrès que ces découvertes représentent pour l'histoire de la fin de l'âge du Bronze en Grèce et en Anatolie a entraîné un véritable enthousiasme dans la communauté scientifique. Enthousiasme d'autant plus grand que la traduction en cours des archives hittites semble promettre d'autres confirmations et que tout reste à démontrer. Les archéologues ont ouvert le débat pour expliquer les raisons d'une guerre de Troie à l'âge du Bronze et surtout pour vérifier à quel niveau archéologique la cité attaquée par les Achéens/Ahhiyawa (si la guerre a eu lieu) pourrait correspondre: Troie VIh, VIIa ou VIIb2. Chacune des solutions a ses implications. Voilà, retracées très sommairement, les lignes de la discussion, de l'émulation et de la compétition scientifique qui a ravivé la question de Troie. Acteur principal de ce débat,

15. Pour un compte-rendu plus précis de ces données, cf. J. Latacz, Troia und Homer: der Weg zur Lösung eines Rätsels et, avec un point de vue critique, M. Benzi, «La guerre fantôme». Voir aussi sur les textes hittites considérés ici J. Freu, "Homère, les Hittites et le pays Ahhiyawa». 
l'homériste bâlois, Joachim Latacz a publié en 2001 un ouvrage qui résume parfaitement l'enjeu des fouilles et qui révèle bien l'espoir de parvenir à la solution: Troie et Homère. Chemins pour la solution d'une vieille énigme (Wege zur Lösung eines alten Rätsels) ${ }^{16}$. Remarquons que le rêve de cette solution est principalement le fait d'une archéologie ou d'une philologie du monde germanique, toujours influencée par Schliemann - je vais y revenir.

\section{Troie, l'archéologue, l'historien et les médias}

C'est donc dans ce contexte d'excitation scientifique que surgit, en 2004, l'énorme vague de publicité faite autour du film Troy de W. Petersen. La promotion du film va passer, une fois n'est pas coutume, par le jugement des universitaires. Partout, en Europe et aux Etats-Unis, historiens et archéologues de la Grèce archaïque se trouvent sollicités pour donner leur avis à la télévision, à la radio ou dans différents journaux. Les plus exposés sont bien sûr les responsables mêmes des fouilles de Troie et du grand projet qui lui est lié. Si bien qu'en juin 2004, lorsque la fièvre retombe, Hans-Günther Jansen exprime son soulagement dans l'éditorial qu'il tient sur le site web du Projet Troie:

Comme il fallait s'y attendre, nous avons dépensé une quantité considérable de temps et d'énergie aux médias. Nous avons été heureux de le faire quand l'intérêt était réel. Mais nous nous réjouissons maintenant de pouvoir nous tourner à nouveau vers les buts premiers de notre travail ${ }^{17}$.

Dans la communauté scientifique, d'autres voix vont se lever pour se fâcher plus nettement contre des médias qui ont trop souvent réduit le débat sur la question homérique à une simple alternative "Homère avait-il raison ou tort?» ${ }^{18}$. Mais archéologues et historiens ont beau jeu de croire à l'indépendance et à l'autonomie de leur entreprise. Entre le projet scientifique et le débat autour du film, l'effet de contamination est

I6. La version française de l'ouvrage n'existe, hélas, pas. La traduction est mienne.

17. Http://www.uni-tuebingen.de//uni/aft/ (juin 2004); je traduis.

I8. Je partage ici entièrement la réaction de F. Montanari, «Les poèmes homériques entre réalité et fiction», p. 10 notamment. 
perceptible. Au niveau commercial d'abord. Si les promoteurs de Troy ont facilement pu gonfler leur publicité en invitant le monde universitaire à cautionner le film, les universitaires n'ont pas manqué d'exploiter à leur tour la publicité du film hollywoodien pour vendre ou faire la publicité de leurs recherches, parfois sous forme de documentaires filmés s'inspirant directement du cinéma, alors même qu'on rappelle le besoin de se démarquer de la conception et de la réalisation du film ${ }^{19}$. Troie est devenu un objet commercial. Mais une fois encore si le produit se vend, c'est que le public en est friand.

Il faut reconnaître que le grand public ne s'intéresse guère au problème historique des relations entre le monde achéen et l'Anatolie à la fin de l'âge du Bronze, quelle que puisse être l'importance de cette question. C'est d'autre chose qu'il s'agit. Qu'on relise les titres des journaux, des documentaires ou des expositions, ils résument l'enjeu de l'intérêt: on veut savoir si «Homère a dit vrai ou non?», si «La guerre de Troie est un mythe ou une réalité?». Comme si cela allait tout expliquer de notre rapport au passé et à la guerre? Comme si l'histoire humaine pouvait se résoudre dans une simple distinction de la part du vrai et du faux? C'est incontestable: beaucoup rêvent d'un Homère qui aurait chanté la vérité. Pourquoi? La question reste ouverte. Peut-être à cause de cette ressemblance ambiguë qui nous lie aux héros homériques? Du moins, peut-on vérifier, dans la presse américaine, le nombre de comparaisons qui retrouvent tel héros derrière tel homme politique ${ }^{20}$.

I9. Voir comme exemple de ce mélange des genres: Myth or Reality, The Truth of Troy, produit par Aidan Laverty, BBC Television, première diffusion sur BBC 2 le 25 mars 2004; Troy. Beyond the Movie, produit par National Geographic et Warner Home Video, 2005. Je fais une place à part au documentaire The Truth About Troy, dans la série Myths of Mankind, produite et dirigée par Roel Oostra, Cresset Communications, 2004, qui fait écho au catalogue de l'exposition Troia: Traum und Wirklichkeit. Et Troie devient aussi un sujet de prédilection dans le monde académique, cf. p. ex. B. S. Strauss, The Trojan war: a new history et C. G. Thomas et C. Conant, The Trojan war.

2o. Voir ici le travail en cours de Gaël Grobéty, cité dans la dernière note. 


\section{Homère et la guerre de Troie: la solution de M. Korfmann}

Qu'ils le veuillent ou non, l'historien et l'archéologue du monde homérique ont été sommés de répondre. Et malgré la prudence de mise, des scénarios d'explication ont été risqués sur ce terrain délicat, à commencer par celui de M. Korfmann lui-même, qui mérite attention vu son autorité. Korfmann est certes prudent; il ne postule pas l'existence d'une guerre de Troie particulière et unique dont le poète garderait la mémoire, mais il cède cependant au rêve d'une poésie ancrée dans la réalité, utilisable par l'archéologue qui, en retour, pourrait prouver le réalisme de certaines descriptions poétiques. Il s'étonne alors de la précision de plusieurs descriptions homériques et souligne certaines données qu'il juge frappantes et non fortuites. Au chant VI de l'Iliade, Andromaque rappelle à Hector l'endroit où les murs de Troie sont le plus faciles à franchir, près du figuier sauvage et donc près de la porte principale. Korfmann constate alors les travaux de renforcement qui ont été effectués, à l'époque du Bronze récent, près de la porte principale (VIU); il observe la fragilité du système défensif à cet endroit ${ }^{21}$. Qu'il s'agisse dans l'Iliade des épithètes qui décrivent Troie, des lieux évoqués, de l'organisation du territoire, de l'emplacement des lavoirs, l'archéologue se plaît à multiplier les rapprochements avec ses découvertes ${ }^{22}$. Pour expliquer cette connaissance poétique des lieux, Korfmann exclut que la tradition orale ait pu transmettre des données si fidèles et il imagine alors un Homère voyageur, qui se serait rendu sur le site de Troie aux alentours de 700 av. J.-C., soit quatre siècles après les faits. A cette époque, il ne reste plus à Troie qu'un sanctuaire, mais les ruines de la prestigieuse Troie VII, les restes des murs, des canalisations, des temples étaient encore visibles. Même Schliemann n'était pas allé si loin, lui qui reconnaissait, dans une lettre à C. Newton, qu'Homère n'avait « jamais vu ni la grande tour d'Ilion, ni le mur divin, ni le palais de Priam " ${ }^{23}$. Mais Korfmann ne doute pas. Son Homère a vu les ruines de la ville et soucieux de situer son récit dans un cadre réaliste, attentif aux données réelles, il aurait travaillé presque comme un

2I. Iliade, VI, 433-434. Voir le commentaire de M. Korfmann, «Ilios, ca 1200 BC Ilion, ca 700 BC», p. 217.

22. Ibid., p. 220-222.

23. Cf. sur cette lettre de Schliemann d'août 1873 les remarques de M. I. Finley, Le monde d'Ulysse, p. 203. 
archéologue ou comme l'auteur d'un roman historique. Quant aux données qui échappent à un ancrage dans la réalité, Korfmann les explique comme le fait d'une coloration mythologique ${ }^{24}$.

On peut critiquer la thèse de Korfmann ${ }^{25}$; la formulation du problème est insidieuse. Dès lors, qu'on cherche à faire la part du mythe et de la réalité, on finit toujours par concéder quelque chose de trop à l'un ou à l'autre. Prenons l'exemple de Mario Benzi qui met son impressionnant savoir au service d'une critique pertinente de la thèse de Korfmann; alors même qu'il répète toute la distance qui sépare la version épique des données archéologiques, il avoue, au vu des données récentes, qu'«on ne résiste pas à la tentation de penser que l'histoire de Pâris et Hélène est peut-être moins invraisemblable qu'on ne le croit et qu'à l'âge du Bronze, et pas seulement à l'âge du Bronze, l'enlèvement d'une princesse royale pouvait déclencher des représailles et même une petite mais féroce guerre entre familles aristocratiques très jalouses de leurs privilèges et de leur honneur " ${ }^{26}$. La suggestion pourrait se transformer en verdict si quelque archive hittite devait évoquer une princesse au nom équivalent à celui d'Hélène. Sur ce point, M. Korfmann semble avoir été plus catégorique quand, dans une déclaration orale, il exclut que l'on puisse "jamais retrouver les restes d'aucun des protagonistes de la guerre de Troie» ${ }^{27}$. Quoi qu'il en soit de ce constat de Korfmann - qui pourrait être un regret -, les résultats des fouilles de Troie ont réveillé chez plusieurs la tentation d'accorder quelque crédit à un bon usage d'Homère pour l'interprétation historique des données archéologiques. Il n'est pas sûr, pourtant, que l'on puisse distinguer si facilement, dans les hexamètres de l'Iliade et de l'Odyssée, entre un souci de réalisme d'une part et l'influence de données mythologiques de l'autre. Homère ne se situe pas au carrefour du réel et de l'imaginaire.

Homère a-t-il vraiment vu au VIII ${ }^{\mathrm{e}}$ siècle ces défauts ou renforcements de fortification que l'archéologue moderne a pu retrouver? A-t-il

24. Ibid., p. 221: "The story of the tepid and cold springs which flow into the Scamander may be due to mythological heightening. " Je reprends ici une observation déjà développée dans D. Bouvier, "Le trône d'Achille», p. 489-492.

25. Par exemple, les points de vue pertinents de F. Montanari, «Les poèmes homériques entre réalité et fiction», p. 19-20 ou M. Benzi, "La guerre fantôme».

26. M. Benzi, «La guerre fantôme», p. 461.

27. Déclaration de M. Korfmann, faite dans le documentaire The Truth About Troy, cité à la note 18 . 
vraiment éprouvé le souci de produire une version réaliste pour ne pas heurter, comme le pense Korfmann, un public qui aurait pu connaître les lieux? Il est absolument impossible de le prouver; mais il me semble impossible d'imaginer un aède travaillant de cette façon ${ }^{28}$. Dans la chaîne des aèdes qui se sont transmis les récits de la geste troyenne, on ne peut pas concevoir un moment de rupture où un aède se mettrait à travailler de façon radicalement différente pour aller visiter le théâtre du récit traditionnel afin de fixer son récit dans une topographie réelle. Une telle attitude, qui peut motiver, au $\mathrm{V}^{\mathrm{e}}$ siècle, l'enquête historique ou géographique, est en contradiction avec la nature même d'une poésie attachée à sa tradition et conçue comme le produit d'une inspiration divine. Il ne s'agit pas ici de nier l'évidence, dans l'Iliade, d'un savoir remarquable sur la cité de Troie et le monde d'alors; mais l'hypothèse d'un Homère voyageur ne peut rendre compte du "pourquoi " de ces connaissances. Il faut plutôt s'étonner de la performance d'une mémoire orale à même de véhiculer un tel savoir au fil des siècles.

$\mathrm{Au}$ terme de cette première partie, il faut bien le relever. La question d'une guerre de Troie entre mythe et réalité est une question sans réponse. Mais c'est surtout un faux problème ou plutôt une question idéologique qui s'inscrit dans la droite ligne d'une longue tradition qui a engendré ses propres contradictions. Moins qu'à l'archéologue, la réponse appartient ici à l'historien des traditions. La Troie d'Homère est une Troie construite par la tradition poétique orale: elle n'est ni fictive, ni réelle; elle est une construction idéologique, produite par une poésie qui décrit le monde en fonction de codes qui lui sont propres ${ }^{29}$.

\section{Histoire d'une question}

L'énigme de la guerre de Troie a fait couler beaucoup d'encre et les solutions que chacun promet si prochainement continueront encore de se multiplier en compliquant la question. Mais, dans cette agitation, personne ne s'est intéressé de près à l'histoire même de cette question. L'objet dépasse les prétentions d'une conférence, il faudrait plusieurs

28. Cf. D. Bouvier, «Le trône d'Achille», p. 491.

29. On pourrait, à défaut d'autres exemples, évoquer la distance entre discours publicitaire et réalité. 
livres; mais puisqu'il s'agit de défendre l'intérêt d'une nouvelle discipline, je tenterai au moins de tracer des lignes. La question du «mythe de la guerre de Troie» est d'abord et avant tout un objet créé par la tradition. Elle n'existe que parce qu'on la fait exister en fonction d'enjeux purement idéologiques. Historiquement, archéologiquement ou épistémologiquement, le problème de la guerre de Troie se pose d'une tout autre façon et ne relève aucunement d'un arbitrage entre le mythe et la réalité. Autant qu'il ignorait notre idée de l'histoire, l'aède homérique ignorait notre idée de la poésie et du mythe.

Les aèdes de l'époque archaïque et les Grecs de l'époque classique auraient été très étonnés de suivre notre débat passionnel sur la réalité de la guerre de Troie. Eux ne se sont jamais posé la question. La guerre de Troie avait eu lieu et ses protagonistes étaient bien les héros évoqués dans l'Iliade et l'Odyssée. Hérodote n'en doute pas une seconde; il en retrouve la preuve tant dans les archives perses que dans le témoignage des prêtres égyptiens qui relatent la guerre de Troie en citant les héros mêmes des poèmes homériques. Hérodote nous donne des dates précises, tant pour la guerre de Troie, un peu plus de huit siècles avant lui, que pour l'époque d'Homère, quatre siècles avant lui ${ }^{30}$. Certes, Hérodote critique la version homérique des faits. Pour lui, Hélène, restée en Egypte, n'est jamais arrivée à Troie. Mais si Hérodote conteste Homère, il n'en suppose pas moins que celui-ci connaissait la vérité et qu'il l'a déformée pour le besoin de sa narration. Hérodote ne pose nulle rupture entre un temps du mythe et un temps de l'histoire. Doutant de la possibilité que certains héros puissent naître de dieux, il déploie une solution ingénieuse en expliquant la nécessité de distinguer deux Héraclès: l'un, connu des Egyptiens, qui fut dieu lui-même et qui vécut plus de $17^{\prime} 000$ ans en arrière, l'autre, né en Grèce, rejeton de parents mortels, qui aurait vécu une génération avant la guerre de Troie, soit neuf siècles avant Hérodote. Le dédoublement ou démultiplication d'un personnage deviendra un outil particulièrement exploité pour résoudre des difficultés chronologiques et concilier l'histoire avec les données traditionnelles.

30. Hérodote, II, 145 et 53 . A ce propos, je donne une bibliographie plus complète dans D. Bouvier, «L'Iliade d'Hérodote». 
On comptera dans certains dictionnaires du XVII ${ }^{\mathrm{e}}$ siècle jusqu'à quinze ou vingt Héraclès ${ }^{31}$.

Et c'est bien parce que l'Iliade traite de l'histoire qu'elle peut être critiquée. Thucydide a une approche analogue. La guerre de Troie a bien eu lieu et Homère reste son meilleur témoin, même s'il faut faire la part de l'exagération et de l'amplification poétique ${ }^{32}$. Au III' siècle av. J.-C. encore, lorsque Eratosthène élabore son système chronologique pour dater les principaux événements de l'histoire grecque, il choisit, comme point de départ et premier repère sûr, la chute de Troie ${ }^{33}$. La guerre de Troie n'est pas seulement un événement historique incontestable, elle est ce moment à partir duquel on peut calculer des écarts et construire la chronologie des événements. D'après le calcul d'Eratosthène, Troie serait tombée en 1184-1183, soit 408 ans avant la première olympiade. Avançons dans le temps et intéressons-nous à un géographe. Au tout début de notre ère, Strabon défend un Homère en qui il reconnaît le premier géographe. En maints endroits, il insiste sur la précision des descriptions homériques ${ }^{34}$. "Partir d'un fait dépourvu de vérité, expliquet-il, n'est pas du tout dans le style d'Homère» ${ }^{35}$. Sa méthode est autre: au fait réel, Homère ajoute simplement, explique Strabon, l'élément du fabuleux ${ }^{36}$. Descendons encore dans le temps pour rencontrer au XII siècle la figure d'Eustathe, grammairien byzantin et archevêque de Thessalonique, à qui l'on doit un vaste commentaire des poèmes homériques qui veut justifier l'intérêt d'Homère aux yeux du christianisme d'Orient. L'archevêque constate qu'Homère, je le cite, déroule son récit

3I. Sur ce dédoublement d'Héraclès chez Hérodote, cf. D. Bouvier, «Memoria epica e memoria storica in Grecia antiqua».

32. Thucydide, I, 211 et 22 4. Cf. C. Calame, Mythe et histoire dans l'Antiquité grecque, p. 28-32.

33. W. Burkert, "Lydia between East and West or How to Date the Trojan War». Eratosthène place l'acmè d'Homère vers 944-943.

34. Strabon, XIII, 15.

35. Strabon, I, 29.

36. Strabon, I, 2 40. Cf. ici Ch. Grell, "Homère géographe au XVIII ${ }^{e}$ siècle" et eadem, "Troie et la Troade de la Renaissance à Schliemann». Sur Strabon lecteur d'Homère, cf. C. Calame, Mythe et histoire dans l'Antiquité grecque, p. 164-165 et A. Trachsel, «Le géographe Eratosthène contre Homère: un choix de Strabon?». 
"en accord avec les faits historiques» ( en l'entrelaçant de mythes ${ }^{37}$. C'est ici l'idée de Strabon qui est reprise.

La guerre de Troie est donc un fait historique unanimement reconnu par les Anciens; mais dès Hérodote, le germe d'une dichotomie entre réalité et exagération poétique est présent. Homère aurait mêlé du fabuleux à du réel. Cette vision va demeurer. Elle nie complètement le fait que les aèdes homériques ignoraient aussi bien les concepts de vérité historique que d'exagération fabuleuse. Ils perpétuaient l'histoire des dieux et des héros selon une perspective épique, qui avaient ses propres règles et exigences. Homère n'était pas tantôt poète, tantôt historien. Il ne mêlait pas mythe et réalité, mais avait sa façon d'appréhender le passé. C'est faute d'admettre ce point que l'on bascule dans l'énigme insoluble de Troie, mythe ou réalité. La part du mensonge et de la vérité ne se fait pas chez Homère au nom d'une distinction entre mythe et histoire, au sens où nous l'entendons ${ }^{38}$.

Quittons l'Orient et le point de vue grec. Dans l'Empire romain d'Occident et durant tout le Moyen Age, Troie fascine et obsède. Il faut rappeler que l'histoire troyenne était alors transmise non pas à partir d'Homère, qui n'avait pas été traduit en latin et qu'on ne savait plus lire, mais à partir surtout d'un livre singulier: le De excidio Trojae, livre qui se présente comme l'œuvre de Darès, nom d'un prêtre troyen dans l'Iliade ${ }^{39}$ et présenté comme un rescapé de la guerre de Troie. Sa version des faits, qui deviendra une référence majeure au Moyen Age, est entièrement favorable aux Troyens, ancêtres de Rome ${ }^{40}$, tandis que les Grecs sont peints comme des guerriers brutaux et sans respect.

Mais les Romains ne sont pas seuls à se dire descendants des Troyens: les Francs, les Normands, les Burgondes, les Germains en font autant. Pour ces peuples, puisque le modèle existe, l'occasion est trop belle de pouvoir revendiquer une origine qui les met sur le même plan que les Grecs et les Romains, modèles incontestés de la culture occidentale.

37. Eustathe, Commentaire à l'Odyssée, I, 1. Je reprends ici la traduction donnée dans l'article de F. Montanari, "Les poèmes homériques entre réalité et fiction", p. 12.

38. Comme le pense Strabon (I, 240 ) et comme l'affirme encore plus un traducteur de Strabon comme Amédée Tardieu en 1867. Ce point reste à développer.

39. Iliade, $\mathrm{V}, 9-11$.

40. Sur le statut de la référence à Troie chez les Grecs et les Romains de l'époque impériale, je renvoie à la riche étude de Ch. Bréchet, "Les Grecs de l'époque impériale et l'origine troyenne de Rome». 
«L'opération visait à valoriser et à légitimer, d'une manière symbolique, l'apparition de la nouvelle puissance, sortie de l'énorme chaos qui avait marqué la fin de l'Empire romain d'Occident. " ${ }^{41}$ Troie devient le lieu d'origine d'une ancienne communauté dispersée : elle permet de tisser les liens qui peuvent unifier les différents états et royaumes d'Europe. Dans ses Chroniques du monde, parues à la fin du XV siècle, Hartmann Schedel dessine en détail ces arbres généalogiques qui font de Troie le berceau commun de la royauté européenne ${ }^{42}$.

Vers 1300, le dominicain milanais Galvaneus Flamma dressait une liste de cités européennes fondées par des Troyens: Pise, Gênes, Palerme, Messine, Brindisi, Tarente, Capoue, Salerne, Vérone, auxquelles il ajoute Toulouse, Barcelone, Tolède, Ségovie, Cologne. Mais encore, certains revendiquaient l'origine troyenne de Paris et de Londres ${ }^{43}$. Et même, Genève, selon la Chronologie du Païs-de-Vaud (Lyon, 1614), revendiquait une origine troyenne par l'intermédiaire d'un certain Lemannus, fils de Pâris, qui servit d'éponyme du lac Léman ${ }^{44}$. D'autres cités se disaient fondées par des Grecs, comme Lisbonne par Ulysse.

Il y a de quoi s'embrouiller dans cette histoire où tout se mêle et où chacun descend des Troyens ou des Achéens. Si les croisés affirment qu'ils veulent venger les Grecs et repousser les musulmans, Mehmed II se rendant sur le site de Troie, neuf ans après la prise de Constantinople, déclare, d'après le chroniqueur grec Critoboulos d'Imbros, qu'il lui est revenu, à lui, de venger les Troyens ${ }^{45}$. Déclaration surprenante! Mais

4I. J. Poucet, «Le mythe de l'origine troyenne au Moyen âge et à la Renaissance», chapitre «Les Francs, les Français et la France».

42. Sur la chronique d'Harmann Schedel, cf. H. Brunner, «"Die in jeder Hinsicht schönste und beste Stadt”. Deutsche Troialiteratur des Spätmittelalters und der Frühen Neuzeit».

43. M. Borgolte, "Europas Geschichten und Troia. Der Mythos im Mittelalter", p. 192. Voir aussi H. Buchthal, Historia Troiana: studies in the history of mediaeval secular illustration.

44. «Après la destruction de Troye, comme Francus, Enée, Antenor, \& plusieurs autres Troyens allerent habiter d'autres Terres; de même Lemannus fils de Pâris vint en ces quartiers, \& s'en étant emparé imposa son nom au Lac Leman». Sur ce texte et sa réfutation par Jacob Spon, cf. J. Poucet, «Le mythe de l'origine troyenne au Moyen âge et à la Renaissance».

45. P. Vidal-Naquet, "L'Iliade sans travesti», p. 5-7. Sur cette revendication de Mehmed II, cf. G. Veinstein, «Histoire turque et ottomane», qui précise: "Vengeur des Troyens, le Turc n'est néanmoins pas présenté ici comme leur descendant, ainsi que le voudront certains humanistes contemporains, en jouant sur la quasi-homonymie entre 
il faut rappeler que les Turcs aussi s'étaient inventé un héros fondateur, Turcus, tout droit venu de Troie. Les humanistes byzantins du $\mathrm{XIV}^{\mathrm{e}}$ siècle enrichissaient la démonstration par l'étymologie en dérivant le nom des Turcs de celui du héros Teucros, héros fondateur de Troie et éponyme des Teucroi, nom donné aux Troyens dès Hérodote. Dans ses Essais, admirant le génie d'Homère, Montaigne évoque une lettre que Mehmed II ("Mahumet, second de ce nom, Empereur des Turcs») aurait écrite au pape Pie II pour s'étonner de l'opposition de Rome à sa conquête:

Je m'estonne comment les Italiens se bandent contre moy, attendu que nous avons nostre origine commune des Troyens: et que j'ay comme eux interest de venger le sang d'Hector sur les Grecs, lesquels ils vont favorisant contre moy ${ }^{46}$.

A la Renaissance, la référence à la guerre de Troie aurait pu aussi bien servir «à sceller une alliance entre François I ${ }^{\text {er }}$ et Soliman le Magnifique» ${ }^{47}$ qu'à amplifier la rivalité entre les Irlandais, perçus comme les descendants des Achéens, et les Anglais qui disaient descendre des Troyens par l'intermédiaire de Brutus, éponyme des Britanniques.

Si les choses sont si compliquées, c'est aussi parce que la guerre de Troie est un double paradigme: Homère a chanté, dans la rencontre d'Achille et Priam, le respect réciproque de deux adversaires, parlant la même langue, partageant même culture et mêmes dieux. Tranchant avec la vision d'Homère, Hérodote est le premier, à notre connaissance, à décrire la guerre de Troie comme une guerre des Grecs rassemblés contre les Barbares, définis comme autres, parlant une autre langue. La guerre de Troie peut fournir le modèle d'un conflit soit interne, soit externe à l'Europe. C'est le paradoxe aussi de Mehmed II, conquérant de Constantinople, qui cherchait également à être un légitime successeur de l'Empire romain. A l'aventurier florentin, Benedetto Dei qu'il fera venir à sa cour, en même temps que des artistes comme Bellini ou Philarète, le sultan expliquait son souci de surpasser «tout à la fois Alexandre et

Teucri et Turci (cf. A. Pertusi, «I primi studi in Occidente sull'origine et la potenza dei Turchi»).» Cf. aussi S. Yerasimos, "La paix de Troie aura-t-elle lieu?». 46. Montaigne, Essais, II, 36 (chap. "Des plus excellens hommes»). 47. Pour reprendre la formule de P. Vidal-Naquet, «L'Iliade sans travesti», p. 7. 
Xerxès » ${ }^{48}$. C'est une évidence, mais l'histoire de Troie n'aurait pas été exploitée à ce point si son historicité n'avait pas été reconnue de tous ${ }^{49}$. Les grandes familles d'Europe ont besoin, pour asseoir leur autorité généalogique, que les héros aient existé. Pourquoi sont-ils devenus légendaires ensuite?

\section{Les héros homériques au Siècle des Lumières}

Le Siècle des Lumières va entreprendre une critique plus systématique et radicale. La noblesse devra avouer les manipulations de ses reconstructions généalogiques. Mais curieusement - ou logiquement - personne, dans ce siècle si critique, ne va remettre en cause l'historicité ni de la guerre de Troie, ni de ses héros. Examinons le verdict des dictionnaires qui fleurissent alors et deviennent les fleurons d'un savoir qu'on n'a cesse de vouloir recenser. Remontons, avant même le XVIII ${ }^{\mathrm{e}}$ siècle, jusqu'au Grand Dictionnaire historique de Louis Moréri qui, en 1683, inventorie aussi, comme l'annonce la page de titre, l'histoire fabuleuse des dieux et des héros de l'Antiquité. Les principaux héros de la guerre de Troie y ont leur article; leur historicité n'est pas mise en cause, même si la présentation fait parfois la part de l'historique et du fabuleux.

Ainsi pour Agamemnon, Moréri rappelle qu'il «étoit Roy d'Argos et de Mycene, qu'il fut élu General de l'armée des Grecs contre les Troyens, $\&$ donna des marques de grand courage, pendant le temps de ce siège si fameux». L'article résume ensuite les données qui viennent de l'Iliade: «Les Poëtes ont soutenu qu'Achille obligea Agamemnon de luy rendre Briséis, qu'il luy avoit enlevée»; mais la distance est prise avec cette version: "quoi qu'il en soit de tous ces contes, il faut ajouter qu'etant de retour dans ses Etats, il fut assassiné par Egysthe fils de Plisthène».

48. M. Philippides, Mehmed II the Conqueror and the fall of the Franco-Byzantine Levant to the Ottoman Turks, p. 141.

49. Dès le XVI ${ }^{\mathrm{e}}$ siècle, des voix s'étaient élevées pour dénoncer ces manipulations de l'histoire. Le juriste Etienne Pasquier (1529-1615), un des esprits les plus ouverts de son temps, ironisait sur cette "grande merveille» qui voyait s'accorder chaque nation «à tirer son ancien estoc de la destruction de Troie» (E. Pasquier, Euvres choisies d'Etienne Pasquier, t. I, p. 47). Et il dénonce la mémoire des hommes «évanouie, ou convertie en belles fables et frivoles" (ibid., p. 8). 
Hélène, de son côté, est définie comme une fille de Tyndare. Son ascendance divine n'est pas évoquée et sa beauté est mentionnée comme cause de deux enlèvements et comme source de la guerre de Troie:

Elle avoit tant de beauté qu'on ne la pouvoit voir sans l'aimer. Thésée l'enleva l'an 2855 du monde. Pâris, fils de Priam Roy de Troye la ravit encore l'an $2860 \&$ cet enlèvement fut le sujet de la guerre de dix ans des Grecs contre les Troyens qui causa la ruine de cette ville.

On retrouve ces dates à l'article consacré à la cité de Troie où la dynastie des rois troyens est donnée:

Troie, ville de l'Asie Mineure dans la Phrygie. On estime que Dardanus, venu ou de Candie ou d'Italie bâtit cette ville, \& fut le premier Roy de ce Royaume des Troïens. Ce fut vers l'an 2574 du Monde. Ses successeurs ont été Erichtonius, Tros, Ilus, Laomédon, \& Priam. Sous le règne de ce dernier, la ville fut brûlée par les Grecs, l'an 1184 avant J.C., après un siège de dix ans, vers l'an 2870 du Monde.

Une décennie plus tard paraît le Dictionnaire historique et critique de Pierre Bayle, conçu au départ pour corriger et critiquer le Grand dictionnaire historique de Moréri. Le travail de Bayle prépare le Siècle des Lumières. Parmi les héros homériques peu présents, il consacre à Achille et à Hélène deux articles de fond où les notes qui développent des détails curieux constituent l'essentiel. Bayle discute longuement, comme il le fait aussi pour Héraclès, les inconséquences du régime alimentaire d'Achille: les poètes montraient Chiron nourrissant son protégé avec de la moelle de lion. L'article consacré à Hélène se plaît lui aussi à dénoncer les inconséquences des données mythologiques qui, si Hélène a été enlevée deux fois, lui donneraient l'âge de soixante ans au terme de la guerre de Troie, sinon cent ans. Quelle armée, interroge Bayle, se serait jamais dérangée pour une femme si âgée dont la beauté devait être bien flétrie. Mais Bayle ne va pas jusqu'à contester l'existence de ces figures. Il rappelle comme un fait acquis que, malgré sa vie déréglée, Hélène reçut des honneurs divins après sa mort et il récuse ceux qui la disent née d'un œuf.

Au milieu du XVIII ${ }^{\mathrm{e}}$ siècle, l'existence de manuels qui s'intitulent explicitement Dictionnaires de mythologie est un fait confirmé. A cette époque-là, le terme de "mythe» est encore loin de faire son entrée dans les dictionnaires de noms communs. En 1727 paraît la première édition du Dictionnaire abrégé de la fable de Pierre Chompré (dont Victor Hugo 
disait ne jamais se séparer), en 1745 celle du Dictionnaire de mythologie pour l'intelligence des poètes, de l'histoire fabuleuse et des monuments historiques de l'abbé André de Claustre, très vite suivi en 1750 par le Dictionnaire abrégé de la fable ou de la mythologie pour l'intelligence des poètes de David Etienne Choffin. Ces manuels répondent à un besoin: le XVIII ${ }^{\mathrm{e}}$ siècle est envahi de mythologie. La référence aux dieux et aux héros antiques est un fait de culture et il faut instruire les jeunes élèves. Du côté de l'Eglise, on s'inquiète. L'abbé Rollin, dans son traité sur la Manière d'enseigner et d'étudier les Belles Lettres paru en 1732, appelle tout haut la rédaction d'ouvrages susceptibles de guider le «jeune enfant chrétien" dans le labyrinthe d'une mythologie où tout n'est pas beau à voir ni à entendre: Mars adultère, Vénus prostituée, Junon incestueuse et Jupiter souillé d'autres crimes plus honteux encore. On est rassuré: les manuels qui paraissent vont présenter des héros convenables. Dans le Dictionnaire abrégé de la fable ou de la mythologie de Choffin, paru en 1750, Achille est présenté comme un roi de Thessalie et il ne mange sa moelle de lion que dans une version allégorique de la fable qui veut nous instruire sur son courage pareil à celui du roi des animaux. Agamemnon, roi d'Argos et de Mycènes, n'est plus fils d'Atrée mais du fameux Pélops. Bon père de quatre filles même s'il dût en sacrifier une pour les besoins de l'Etat. Et Hélène, belle et tout à fait humaine, est fille de Tyndare, sans qu'il ne soit plus guère question d'un ouf et d'amour avec un cygne. La mythologie est ici épurée de sa part scandaleuse: la rationalisation du mythe obéit au critère moral. Remarquons que Chompré et Choffin sont aussi, chacun, auteurs d'un Dictionnaire abrégé de la Bible.

L'apparition de ces manuels de mythologie bon ton n'entraîne aucunement une quelconque mise en doute de l'historicité des personnages présentés, au contraire. Dans ces manuels, rien d'étonnant non plus à trouver aux côtés d'Achille et d'Agamemon, Alexandre le Grand, "qui eut la manie de vouloir aussi passer pour un Dieu».

Que se passe-t-il dans l'Encyclopédie de d'Alembert et Diderot qui s'est appuyée sur ces ouvrages? On sait que les rédacteurs de l'Encyclopédie avaient initialement décidé d'exclure les noms propres de personnes historiques mais une exception fut faite pour les noms bibliques et mythologiques, qui constituaient, à eux seuls, un domaine de savoir. Malgré l'existence des dictionnaires de la fable et de la mythologie qui ont organisé la matière, les rédacteurs de l'Encyclopédie sont loin de soustraire à l'histoire les héros antiques auxquels ils consacrent un article. 
Référons-nous aux indications en italiques, mises entre parenthèses, qui classent chaque article dans une rubrique thématique. Si les dieux (Apollon, Artémis, Diane, Jupiter, Minerve...) et les êtres merveilleux (Centaures) sont bien classés dans le domaine de la mythologie (Myth.), dans le cas des héros, le classement reste indécis. Hélène est rattachée à l'histoire ancienne (Hist. anc.), Hercule à la mythologie et à la littérature (Mytholog. \& Littérat.), Médée à l'histoire grecque et à la mythologie (Hist. grecq. \& Mythol.). Les étiquettes sont ici un témoin précieux pour comprendre que la Mythologie n'est encore séparée ni de l'Histoire ancienne ni de la Littérature. Branche reconnue des Belles-Lettres, elle ne définit pas une discipline autonome et spécifique mais recouvre un ensemble de connaissances qui renvoie aussi bien à l'étude de la Théologie qu'à celle de l'Histoire. A l'article "mythologie», le Chevalier de Jaucourt ne postule l'intérêt supérieur des fables historiques que parce qu'il croit à l'historicité des héros. Et l'étude des volumes du Supplément confirme cette impression: Turpin classe les différents héros de l'Iliade aussi bien sous l'étiquette d'Histoire poétique (Ajax) que sous celle d'Histoire ancienne ou Mythologie (Agamemnon) tout en donnant des dates ${ }^{50}$.

Ni l'existence du Dictionnaire de mythologie qui découpe son savoir en le recensant, ni les étiquettes de l'Encyclopédie n'entraînent donc une mise en cause quelconque de la dimension historique des héros. Qu’il suffise de citer l'article consacré à la cité de Troie. Si son rédacteur, le Chevalier de Jaucourt, invite à «se souvenir toujours des mille fables mêlées dans tout ce que les poëtes nous disent du siège de Troie", il ne critique vraiment que l'épisode du cheval de bois. Surtout, il livre des dates sur la fondation de la ville, sur les règnes successifs de ses rois, sur sa destruction "dix après l'enlèvement d'Hélène, en l'an du monde 2820, 1184 ans avant l'ere vulgaire, \& donc 431 ans avant la fondation de Rome». L'article évoque aussi assez longuement l'état des ruines de la ville, tout en évoquant les positions de Pierre Belon du Mans et Pietro della Valle qui les identifiaient aux restes de la Troie d'Homère, tandis que M. Spon plus récemment y voyait des ruines de l'époque romaine. Le débat sur les ruines de Troie est bien engagé mais personne ne conteste la réalité de la guerre de Troie. Voltaire lui-même le constate dans ses lettres philosophiques:

50. Je reprends ici une argumentation développée dans D. Bouvier, «Le héros grec au pays des dictionnaires". 
Jamais aucun écrivain n'a révoqué en doute la guerre de Troie. La fiction de la pomme d'or ne détruit pas la vérité du fond du sujet. L'ampoule apportée par une colombe, et l'oriflamme par un ange, n’empêchent pas que Clovis n'ait en effet régné en France ${ }^{51}$.

On pourrait ici ouvrir une autre voie et regarder le point de vue des voyageurs romantiques. On rêve alors d'un Homère poète des origines, porte-parole d'une langue sans travesti, immédiate à la nature. Pour les voyageurs, surtout anglais, qui vont vers l'Orient et passent par Troie, Homère est le témoin direct et crédible du monde fascinant qu'il décrit. Parce qu'il ignorait l'abstraction et les habiles constructions de la pensée, le premier poète grec se trouvait incapable d'altérer les réalités qu'il voyait. En Turquie, où elle avait suivi son époux devenu ambassadeur à Constantinople, Lady Mary Montagu visite Troie et, dans une lettre à Alexander Pope, illustre traducteur de l'Iliade et son admirateur avant leur querelle, elle écrit en 1717 :

Devant ces paysages et ces rivières célèbres, j'admirais l'exactitude d'Homère que j'avais en main. Presque toutes les épithètes dont il qualifie les montagnes et les plaines sont exactes ${ }^{52}$.

Peu après elle, Thomas Blackwell et surtout Robert Wood tiendront le même propos. Se rendant à Troie en 1734, Wood publia son Essai sur le génie d'Homère en 1775. Il y explique comment, arrivant à Troie, il s'émerveilla de la qualité des descriptions d'Homère:

Tous ses tableaux répondent plus à l'état actuel du pays, qu'on n'auroit lieu de l'attendre. Les rochers, les collines, les vallons, les promontoires qu'il décrit attestent encore aujourd'hui son exactitude ${ }^{53}$.

5I. Note de Voltaire du 10 mai 1738; cette remarque a été intégrée dans l'édition des Euvres complètes de Voltaire de 1742 où elle est ajoutée à la vingt-cinquième lettre; cf. R. Naves, Voltaire, Lettres philosophiques, lettre XXV, remarque 71, p. 276.

52. Lettre 44; cf. M. W. Montagu, L'Islam au péril des femmes, p. 220. Sur les voyageurs romantiques visitant la plaine de Troie, cf. Ch. Grell, "Homère géographe au XVIII ${ }^{\mathrm{e}}$ siècle»; A. Trachsel, La Troade: un paysage et son héritage littéraire; les commentaires antiques sur la Troade, leur genèse et leur influence et D. Bouvier, "Une archéologie du paysage à l'envers ou les romantiques dans la plaine de Troie».

53. R. Wood, Essai sur le génie d'Homère avec l'état actuel de la Troade comparé à son état ancien, p. 70. 
Depuis Strabon déjà, l'Homère excellent géographe suppose l'Homère historien. Wood appartient à un courant que Schliemann et Korfmann prolongeront à leur façon. Et le parallélisme devient surprenant quand Wood constate que le «rapport est si naturel \& si plausible entre la scène $\&$ l'action de l'Iliade" que cela porte "à croire qu'il [Homère] a été témoin oculaire de l'une, \& que les traditions de son tems lui ont fourni l'autre». Et de poursuivre:

Comme il vivoit dans le voisinage de Troie, cette conjecture devient plus probable, car il eut occasion de bien connoître les bords du Simoïs $\&$ du Scamandre, \& d'apprendre de ceux qui avoient vu le siège \& qui s'étaient signalés dans les combats, ou du moins de leurs enfans, les circonstances $\&$ les détails de leurs exploits les plus renommés de la guerre $^{54}$.

C'est exactement, deux cent trente ans plus tôt, la position de Korfmann: Homère a visité les lieux où la guerre s'était faite. Ladéquation entre le paysage et le poème est telle qu'Homère a dû voir Troie. Peintre d'un âge d'avant la raison ou poète épris de réalisme, Homère est un témoin digne de foi. Remarquons aussi que le travail de Wood répond, à distance, aux détracteurs d'Homère qui, après la Querelle des Anciens et des Modernes, avait mis en doute l'unité de l'Iliade et l'existence d'un poète unique. La précision des descriptions topographiques de l'Iliade est là pour prouver le génie d'Homère.

Inutile de dire qu'à chaque fois les tableaux homériques si précis sont décrits autrement et quaucun de ces fanatiques de l'exactitude homérique n'est d'accord avec l'autre sur les lieux et les détails des emplacements.

7. Quand les héros homériques deviennent des êtres de légende au XIX ${ }^{e}$ siècle

Les héros homériques continuent tout au long du XIX ${ }^{\mathrm{e}}$ siècle d'habiter aussi bien les pages des manuels de mythologie que celles des Dictionnaires historiques ou autres biographies universelles. En 1856, le Dictionnaire universel d'histoire et de géographie de M.-N. Bouillet présente Achille, Agamemnon et Hélène comme des personnages

54. Ibid., p. 159. 
historiques, sans omettre de nous livrer les dates de leurs règnes. La situation va changer dans la deuxième partie du XIX ${ }^{\mathrm{e}}$ siècle. Ouvrons le Grand Dictionnaire Universel du XIX ${ }^{e}$ siècle paru entre 1866 et 1879. Les héros basculent dans la légende et les dates disparaissent. Avant d'être des rois ou des reines, Achille, Agamemnon et Hélène sont d'abord des héros de l'Iliade dont on précise qu'ils "appartiennent plus à la légende païenne qu'à l'histoire». Leurs ascendances divines, discrètes auparavant, sont mises en avant. Un siècle plus tard, la rupture est consommée: dans le Grand Larousse encyclopédique de 1964, les héros homériques sont devenus définitivement des êtres légendaires. Hélène n'est plus présentée comme la fille de Tyndare ou même de Zeus, mais comme née d'un œuf. Devenu figure légendaire, mythique et fabuleuse, le héros quitte logiquement les manuels d'histoire; on ne s'étonnera guère de leur absence dans le Dictionnaire des personnages historiques de tous les temps de M. Mourre (Paris, Bordas, 1972) ou dans le Dictionnaire des personnages historiques de J.-L. Voisin (Paris, Livre de Poche, 1995). A la fin du XXe siècle, le héros ne peut exister dans deux camps à la fois. Sa présence dans le dictionnaire de mythologie exclut désormais son appartenance à l'histoire.

Que s'est-il passé qui a entraîné ce processus de "déshistorisisation" des héros antiques? On évoquera bien sûr l'émergence, au milieu du $\mathrm{XIX}^{\mathrm{e}}$ siècle, de la mythologie comme science des mythes et nouvelle discipline académique dans les universités. Travaillant sur l'imaginaire des différentes religions, s'interrogeant sur l'universalité des mythes ou des structures du mythe, assimilant la poésie homérique à un recueil de mythologie, les nouvelles lignes de recherche s'intéressent aux héros comme à des figures exemplaires et archétypiques, hors de toute histoire. Et si les héros sont des êtres légendaires, la guerre de Troie ne peut être qu'un mythe.

Il y a, toutefois, toujours des voyageurs qui vont à Troie et qui postulent la fidélité des descriptions d'Homère. C'est le cas en 1867 de Georges Nicolaïdès qui, écrivant une topographie de l'Iliade, s'exclame: «si la Troade venait à disparaître entièrement, on pourrait avec la seule Iliade la dessiner et la recomposer ${ }^{55}$. Mais le plus célèbre de ces voyageurs est bien sûr Schliemann, arrivé à Troie en 1870. Alors que les chaires de mythologie se répandent partout en Europe, il est le dernier à

55. G. Nicolaïdès, Topographie et plan stratégique de l'Iliade, avec une carte topographique et stratégique, p. 4. 
croire si fort qu'un archéologue peut retrouver la tombe d'Agamemnon ou le trésor de Priam. Cent trente ans plus tard, la question est relancée par la présence, dans les textes hittites, de noms assimilables à ceux des héros homériques. Et certains pensent déjà que l'Alaksandu roi de Wilusa est bien le modèle qui a pu inspirer le personnage d'Alexandre, un Alexandre qui pourrait alors rejoindre les dictionnaires historiques qu'il avait quittés. Historique, puis mythique, le héros pourrait redevenir historique.

8. Quand l'archéologue de Troie devient un allié d'Hollywood et du gouvernement turc

Homère historien? Homère poète? Chaque époque choisit l'Homère qui l'intéresse. Et le débat se réduit souvent à cette alternative. En ce début de XXI ${ }^{\mathrm{e}}$ siècle, le cinéma hollywoodien a donné à l'archéologie une caution dont on ne sera finalement pas si surpris. Ce n'est sans doute pas un hasard si la guerre de Troie est redevenue un sujet d'actualité. Il n'est du moins pas sans intérêt de constater que la Turquie actuelle s'est grandement réjouie des fouilles et des découvertes de Manfred Korfmann. Le gouvernement turc a tenu à honorer l'archéologue en lui offrant la citoyenneté turque et le nom d'Osman, fondateur de la dynastie ottomane: c'est un honneur exceptionnel dans un pays où les lois sont très restrictives dans le domaine de la citoyenneté. L'archéologue allemand est ainsi devenu Manfred Osman Korfmann. Dans le journal Radikal, qui est un journal plutôt ouvert, favorable à la cause kurde, un intellectuel comme Haluk Sahin, qui s'est récemment engagé pour ouvrir le débat sur la question du génocide arménien en Turquie, n’hésite pas à écrire que "le rôle des Turcs aujourd'hui est d'être dans le rôle historique des Troyens " ${ }^{56}$. Phrase compliquée mais qui perpétue, bien après Mehmed II, le désir des Turcs d'être liés à la culture occidentale. Prétention que l'Amérique a bien relevée quand à son tour elle a cherché,

56. «Günümüz Türklerinin tarihsel rol olarak Troyalı olduğuna ise hiç kuşkum yok», citation tirée de l'article "Troyalılar Türk müydü?», in Radikal, 16 mai 2004 (partie 1) et 23 mai 2004 (partie 2) (traduction de H. L. Kieser) ; cf. la version informatique sur: http://www.radikal.com.tr/haber.php?haberno=117193 et http://www.radikal.com.tr/haber.php?haberno=116492. 
pour ses missions militaires, des bases aux portes de l'Orient: l'Irak était le nouvel ennemi absolu ${ }^{57}$.

En décembre 2001, trois mois après les attentats du 11 septembre, la Warner Bros (la société qui a diffusé le film Troy) offrait à Brad Pitt (futur Achille) et à Angelina Jolie un voyage en Turquie pour aller réconforter les soldats américains et britanniques stationnés dans des bases secrètes en vue de raids contre l'Irak; le cinéma rencontrait l'actualité. La presse invoque alors l'esprit de "collaboration" que les patrons d'Hollywood souhaitent instaurer avec le gouvernement américain, et vice-versa. Il se trouve aussi que cette mission morale de Brad Pitt et d'Angelina Jolie coïncide avec le lancement, le même mois, de leur dernier film d'alors, Ocean Eleven, produit par un ami de longue date du président George Bush, Jerry Weintraub.

Trois ans plus tard et alors que le gouvernement américain a répété à Hollywood ses consignes sur l'image de l'Islam dans les films à venir et son souci d'éviter les amalgames négatifs, la Warner Bros accepte de financer le gros budget du film Troy. Le scénario du film est largement favorable aux Troyens. L'Achille hollywoodien de Troy le dit tout haut: il aurait préféré servir le juste Priam plutôt que le cupide Agamemnon. Ici encore l'exploitation du mythe de la guerre de Troie n'est pas sans intention. A sa façon, Hollywood a compris le parti à tirer d'une image positive revalorisant les Troyens. Le mythe n'est jamais aussi utile que quand il permet de réécrire l'histoire dont le présent a besoin. A distance, le scénario de la guerre de Troie a été refait et a permis, au passage, de faire un clin d'œil à la Turquie.

\author{
David Bouvier \\ Université de Lausanne
}

57. L'influence de l'actualité sur la réception de l'Iliade dans la culture américaine d'aujourd'hui (médias, fiction, travaux scientifiques) fait l'objet d'un travail que mène actuellement sous ma direction Gaël Grobéty; provisoirement, on peut se référer à son article: "La réception de l'Iliade dans la culture américaine contemporaine. L'exemple des articles journalistiques", à paraître dans Langage du pouvoir, pouvoir du langage, éd. G. Gianotti. 


\section{BIBLIOGRAPHIE}

Troia: Traum und Wirklichkeit, [Ausstellung, Stuttgart, Forum der Landesbank Baden-Württemberg, 17. März bis 17. Juni 2001; Braunschweig, Braunschweigisches Landesmuseum und HerzogAnton Ulrich-Museum/Burg Dankwarderode, 14. Juli bis 14. Oktober 2001; Bonn, Kunst und Ausstellungshalle der Bundesrepublik Deutschland, 16. November 2001 bis 17. Februar 2002], Stuttgart, Theiss, 2001.

Benzi, Mario, "La guerre fantôme. La guerre de Troie dans une perspective archéologique", in Reconstruire Troie. Permanences et renaissances d'une cité emblématique, éds Michel Fartzoff, Murielle Faudot, Evelyne Geny et Marie-Rose Guelfucci, Besançon, PUFC, 2009, p. 445-468.

Borgolte, Michael, «Europas Geschichten und Troia. Der Mythos im Mittelalter", in Troia: Traum und Wirklichkeit, Stuttgart, Theiss, 2001, p. 190-203.

Bouvier, David, «Une archéologie du paysage à l'envers ou les romantiques dans la plaine de Troie", Sources-Travaux historiques, 5 (1986), p. 89-91.

—, "Memoria epica e memoria storica in Grecia antica», in L'antico degli antichi, a cura di Guglielmino Cajani e Diego Lanza, Palermo, Palumbo, 2001, p. 31-61.

—, "Des Fontaines de Troie à la prison d'Abou Ghraib», Le Temps, 22 mai 2004.

—, "Le héros grec au pays des dictionnaires", Europe (Historiens de l'Antiquité), 904-905 (2004), p. 254-266.

—, «L'Iliade d'Hérodote», Europe (Historiens de l'Antiquité), 945-946 (2008), p. 74-86.

—, «Le trône d'Achille: représentations de chaises et fauteuils dans l'Iliade: regards philologique et archéologique", in Reconstruire Troie. Permanences et renaissances d'une cité emblématique, éds Michel Fartzoff, Murielle Faudot, Evelyne Geny et MarieRose Guelfucci, Besançon, PUFC, 2009, p. 489-510. 
BrÉchet, Christophe, "Les Grecs de l'époque impériale et l'origine troyenne de Rome", in Reconstruire Troie. Permanences et renaissances d'une cité emblématique, éds Michel Fartzoff, Murielle Faudot, Evelyne Geny et Marie-Rose Guelfucci, Besançon, PUFC, 2009, p. 425-444.

Brunner, Horst, " "Die in jeder Hinsicht schönste und beste Stadt". Deutsche Troialiteratur des Spätmittelalters und der Frühen Neuzeit", in Troia: Traum und Wirklichkeit, Stuttgart, Theiss, 2001, p. 212-225.

BRyCE, Trevor, "An historian's observation on Troy and homeric tradition", in Homère et l'Anatolie, éd. Michel Mazoyer, Paris, L'Harmattan, 2008, p. 31-46.

Buchthal, Hugo, Historia Troiana: studies in the history of mediaeval secular illustration, London/Leiden, Brill, 1971.

Burkert, Walter, «Lydia between East and West or How to Date the Trojan War: A Study in Herodotus", in The Ages of Homer. A Tribute to Emily Townsend Vermeule, eds Jane B. Carter and Sarah P. Morris, Austin, University of Texas Press, 1995, p. 139-148.

Calame, Claude, Mythe et histoire dans l'Antiquité grecque, Lausanne, Payot, 1996.

Crielaard, Jan Paul, "Homer, History and Archaeology: Some Remarks on Date of the Homeric World", in Homeric Questions. Essays in Philology, Ancient History and Archaeology, ed. Jan Paul Crielaard, Amsterdam, J. C. Gieben, 1995, p. 183-200.

Danek, Georg, "The Story of Troy through the Centuries", in Troy: from Homer's Iliad to Hollywood epic, ed. Martin M. Winkler, Malden (MA), Blackwell, 2007, p. 68-84.

Finley, Moses I., Le monde d'Ulysse, nouvelle édition augmentée, Paris, Maspero, 1978.

Freu, Jacques, "Homère, les Hittites et le pays Ahhiyawa», in Homère et l'Anatolie, éd. Michel Mazoyer, Paris, L'Harmattan, 2008, p. 77-106.

Grell, Chantal, "Troie et la Troade de la Renaissance à Schliemann", Journal des Savants (1981), p. 47-76.

—, "Homère géographe au XVIII siècle», in Homère en France après la Querelle (1715-1900), éds Françoise Létoublon et C. VolplilhacAuger, Paris/Genève, Slatkine, 1999, p. 255-264. 
Haw kins, J. David et Easton, Donald F., «A Hieroglyphic Seaal from Troia», Studia Troica, 6 (1998), p. 111-118.

Hertel, Dieter, Troia: Archäologie, Geschichte, Mythos, München, C. H. Beck, 2001.

-, Die Mauern von Troia: Mythos und Geschichte im antiken Ilion, München, C. H. Beck, 2003.

Kolb, Frank, "Troy VI: a trading center and commercial city", American Journal of Archaeology, 108 (2004), p. 577-613.

Korfmann, Manfred, "Ilios, ca 1200 BC - Ilion, ca 700 BC», in Omero tremila anni dopo, a cura di Franco Montanari, Roma, Edizioni di storia e letteratura, 2002, p. 209-226.

Korfmann, Manfred (hrsg.), Troia: Archäologie eines Siedlungshügels und seiner Landschaft, Mainz, Ph. von Zabern, 2006.

Korfmann, Manfred, Frank, Norbert et Mangini, Augusto, «Eingang in die Unterwelt. Die Höhle von Troia une ihre Datierung", in Troia: Archäologie eines Siedlungshügels und seiner Landschaft, hrsg. von Manfred Korfmann, Mainz, Ph. von Zabern, 2006, p. 337-342.

Latacz, Joachim, Troia und Homer: der Weg zur Lösung eines alten Rätsels, München/Berlin, Koehler \& Amelang, 2001.

—, "From Homer's Troy to Petersen's Troy", in Troy: from Homer's Iliad to Hollywood epic, ed. Martin M. Winkler, Malden (MA), Blackwell, 2007, p. 27-42.

Montagu, Mary Wortley, L'islam au péril des femmes: une Anglaise en Turquie au XVIII ${ }^{e}$ siècle, (édition établie par Anne-Marie Moulin et Pierre Chuvin), Paris, La Découverte, 1981.

Montanari, Franco, "Les poèmes homériques entre réalité et fiction", Gaia, 9 (2005), p. 9-24.

—, "Troia omerica e Troia anatolica. L'eterno dubbio tra realta e fantasia», in Troia tra realtà e leggenda, a cura di Gabriele Burzacchini, Parma, Monte Universita Parma, 2005, p. 11-21.

Naves, Raymond, Voltaire. Lettres philosophiques ou lettres anglaises avec le texte complet des remarques sur les pensées de Pascal, Paris, Flammarion, 1979.

Nicolä̈DÈs, Georgios, Topographie et plan stratégique de l'Iliade, avec une carte topographique et stratégique, Paris, Hachette, 1867.

Pasquier, Etienne, Euvres choisies d'Etienne Pasquier, éditées par Léon Feugère, t. I, Paris, 1849 (= Paris, repr. Slatkine, 1968). 
Pertusi, Agostino, «I primi studi in Occidente sull'origine e la potenza dei turchi», Studi veneziani, 12 (1970), p. 468-552.

Phillipides, Marios, Mehmed II the Conqueror and the fall of the FrancoByzantine Levant to the Ottoman Turks: some western views and testimonies, Tempe (Ariz.), Center for Medieval and Renaissance Studies, 2007.

PoucET, Jacques, «Le mythe de l'origine troyenne au Moyen Age et à la Renaissance: un exemple d'idéologie politique», Folia Electronica Classica, 5 (2003) : http://bcs.fltr.ucl.ac.be/fe/05/anthenor02.html.

Poucet, Jacques et Hannick, Jean-Marie, Aux sources de l'antiquité gréco-romaine: guide bibliographique, Bruxelles, Artel, 1997.

Reber, Karl, "Troie. découverte et état actuel des connaissances", in Le cheval de Troie, éd. Danielle van Mal-Maeder, Gollion, Infolio, 2007, p. 1-32.

Solomon, Jon, "Viewing Troy: Authenticity, Criticism, Interpretation", in Troy: from Homer's Iliad to Hollywood epic, ed. Martin M. Winkler, Malden (MA), Blackwell, 2007, p. 85-98.

Strausss, Barry Stuart, The Trojan War: a new history, New York, Simon \& Schuster, 2006.

Thomas, Carol G. et Conant, Craig, The Trojan war, Norman, University of Oklahoma Press, 2007.

Trachsel, Alexandra, La Troade: un paysage et son héritage littéraire; les commentaires antiques sur la Troade, leur genèse et leur influence, Basel, Schwabe, 2007.

—, "Le géographe Eratosthène contre Homère: un choix de Strabon?", in Eratosthène: un athlète du savoir, éds Christophe Cusset et Hélène Frangoulis, Saint-Etienne, Publications de l'Université de Saint-Etienne, 2008, p. 105-119.

Veinstein, Gilles, "Histoire turque et ottomane», Annuaire du Collège de France. Résumé des cours et travaux, 2001-2002, p. 857-872.

Vidal-Naquet, Pierre, "L'Iliade sans travesti ", in Homère. Iliade, Paris, Gallimard, 1975, p. 5-32.

WeIL, Simone, "L'Iliade ou le poème de la force (1940-1941)», in Euvres, édition établie par Florence de Lussy, Paris, Gallimard, 1999, p. 527-552.

Winkler, Martin M. (ed.), Troy: from Homer's Iliad to Hollywood epic, Malden (MA), Blackwell, 2007. 
-, "The Iliad and the Cinema", in Troy: from Homer's Iliad to Hollywood epic, ed. Martin M. Winkler, Malden (MA), Blackwell, 2007, p. 43-67.

-, "The Trojan War on the Screen", in Troy: from Homer's Iliad to Hollywood epic, ed. Martin M. Winkler, Malden (MA), Blackwell, 2007, p. 202-222.

Wood, Robert, Essai sur le génie original d'Homère avec l'état actuel de la Troade comparé à son état ancien, Paris, Frères de Bure, 1777.

Yerasimos, Stéphane, «La paix de Troie aura-t-elle lieu?», Cahiers d'études sur la Méditerranée orientale et le monde turco-iranien, 8 (1989), p. 17-24. 